\title{
MONTEL SUBSPACES OF FRÉCHET SPACES OF MOSCATELLI TYPE
}

\author{
by ANGELA A. ALBANESE $\dagger$
}

(Received 13 May, 1996)

In this note we show that every complemented Montel subspace $F$ of a Fréchet space $E$ of Moscatelli type is isomorphic to $\omega$ or is finite-dimensional; the last case always occurs when $E$ has a continuous norm. To do this, we first study the topology induced by $E$ on its Montel subspaces, extending a result on Fréchet-Montel spaces of Moscatelli type in [4].

We recall that the Fréchet spaces of Moscatelli type were introduced and studied by $J$. Bonet and S. Dierolf in [4]; the general idea behind the construction of such spaces was due to V. B. Moscatelli [7].

The paper has three sections. The first one is devoted to the necessary definitions and preliminaries; in the second we prove our main result and in the third we apply it to some concrete function spaces of Fréchet-Sobolev type.

1. Definitions and preliminaries. Let $(\lambda,\|\|)$ be a normal Banach sequence space, i.e. a Banach space satisfying

(a) $\varphi \subset \lambda \subset \omega$ algebraically and the inclusion $(\lambda,\|\|) \hookrightarrow \omega$ is continuous

(B) $\forall a=\left(a_{k}\right)_{k} \in \lambda, \forall b=\left(b_{k}\right)_{k} \in \omega$ such that $\left|b_{k}\right| \leq\left|a_{k}\right|, \forall k \in \mathbf{N}$, we have $b \in \lambda$ and $\|b\| \leq\|a\|$.

Of course, every projection

$$
s_{n}: \omega \rightarrow \omega, \quad\left(a_{k}\right)_{k} \rightarrow\left(\left(a_{k}\right)_{k \leq n},(0)_{k>n}\right)
$$

onto the first $n$-coordinates induces a \|\| -decreasing endomorphism on $\lambda$. We shall introduce the following property on $(\lambda,\|\|)$ :

$$
\lim _{n \rightarrow \infty}\left\|a-s_{n}(a)\right\|=0, \quad \forall a \in \lambda .
$$

Typical examples of $(\lambda,\|\|)$ are the spaces $\left(l^{p},\|\|_{p}\right)$, where $1 \leq p \leq \infty,\left(c_{0},\|\|\right)$ and their diagonal transforms. In particular, the spaces $\left(l^{p},\|\|\right)$, where $1 \leq p<\infty$, and $\left(c_{0},\|\|\right)$ satisfy $(\varepsilon)$.

Now, let $(\lambda,\|\|)$ be a normal Banach sequence space and let $\left(Y_{k},\|\|_{k}\right)_{k}$ be a sequence of Banach spaces; then the Banach space $\lambda\left(\left(Y_{k},\|\|_{k}\right)_{k}\right)$ is defined as the linear space

$$
\lambda\left(\left(Y_{k},\|\|_{k}\right)_{k}\right)=\left\{\left(y_{k}\right)_{k} \in \prod_{k \in \mathbb{N}} Y_{k}:\left(\left\|y_{k}\right\|_{k}\right)_{k} \in \lambda\right\},
$$

endowed with the norm $\left(y_{k}\right)_{k} \rightarrow\left\|\left(\left\|y_{k}\right\|_{k}\right)_{k}\right\|$.

Let $\left(X_{k},||_{k}\right)_{k}$ be another sequence of Banach spaces and, for every $k \in \mathbf{N}$, let $f_{k}: X_{k} \rightarrow Y_{k}$ be a linear map such that $\left\|f_{k}(x)\right\|_{k} \leq|x|_{k}$, for every $x \in X_{k}$.

Then, following J. Bonet and S. Dierolf [4, Definition 1.3 and Proposition 1.4], we define the Fréchet space $E$ of Moscatelli type with respect to $(\lambda,\|\|),\left(X_{k},||_{k}\right)_{k}$,

$\dagger$ Research supported by the Italian MURST.

Glasgow Math. J. 39 (1997) 345-350. 
$\left(Y_{k},\|\|_{k}\right)_{k}$ and $\left(f_{k}\right)_{k}$ as the space

$$
E=\left(\prod_{k \in \mathbb{N}} X_{k}\right) \cap \lambda\left(\left(Y_{k},\|\|_{k}\right)_{k}\right)=\left\{\left(x_{k}\right)_{k} \in \prod_{k \in \mathbb{N}} X_{k} ;\left(f_{k}\left(x_{k}\right)\right)_{k} \in \lambda\left(\left(Y_{k},\|\|_{k}\right)_{k}\right)\right\}
$$

with the intersection topology given by the sequence of seminorms

where

$$
p_{n}\left(\left(x_{k}\right)_{k}\right)=r_{n}\left(\left(x_{k}\right)_{k}\right)+r\left(\left(x_{k}\right)_{k}\right) \quad\left(\left(x_{k}\right)_{k} \in E\right),
$$

$$
r_{n}\left(\left(x_{k}\right)_{k}\right)=\sup _{k \leq n}\left|x_{k}\right|_{k} \text { and } r\left(\left(x_{k}\right)_{k}\right)=\left\|\left(\left\|f_{k}\left(x_{k}\right)\right\|_{k}\right)_{k}\right\| .
$$

For more about such spaces the reader is referred to, for example, [4] and [7].

Also, we introduce for every $n \in \mathbf{N}$ the following continuous maps

$$
\begin{gathered}
J_{n}: \prod_{k \leq n} X_{k} \rightarrow E,\left(x_{1}, \ldots, x_{n}\right) \rightarrow\left(\left(x_{k}\right)_{k \leq n},(0)_{k>n}\right), \\
S_{n}: E \rightarrow \prod_{k \leq n} X_{k},\left(x_{k}\right)_{k} \rightarrow\left(x_{1}, \ldots, x_{n}\right),
\end{gathered}
$$

where $S_{n}$ is the restriction to $E$ of the canonical projection from $\prod_{k=1}^{\infty} X_{k}$ onto $\prod_{k \leq n} X_{k}$. Clearly, $J_{n}$ is an isomorphism into and $S_{n} J_{n}=I_{\prod_{k \leq n} X_{k}}$, for every $n \in \mathbf{N}$. Hence the map $J_{n} S_{n}$ is a projection from $E$ onto its Banach subspace $J_{n}\left(\prod_{k \leq n} X_{k}\right)$. Moreover, we recall that the countable product space $\prod_{k=1}^{\infty} X_{k}$ can be represented as $\operatorname{proj}_{n}\left(\prod_{k \leq n} X_{k}, S_{n+1, n}\right)$, where $S_{n+1, n}: \prod_{k \leq n+1} X_{k} \rightarrow \prod_{k \leq n} X_{k}$ is the canonical projection $\left(x_{1}, \ldots, x_{n}, x_{n+1}\right) \rightarrow\left(x_{1}, \ldots, x_{n}\right)$.

We shall use standard terminology for the theory of Fréchet spaces, as in [5]. In particular, for two Fréchet spaces $F$ and $G$, we shall write $F=G$ to mean that $F$ is topologically isomorphic to $G$.

2. Montel subspaces of Fréchet spaces of Moscatelli type. For the sequel, $E$ always denotes the Fréchet space of Moscatelli type with respect to $(\lambda,\|\|),\left(X_{k},||_{k}\right),\left(Y_{k},\|\|_{k}\right)$ and $f_{k}: X_{k} \rightarrow Y_{k}$, with $\left\|f_{k}(x)\right\|_{k} \leq|x|_{k}$ for every $x \in X_{k}$. We shall assume that $(\lambda,\|\|)$ satisfies the property $(\varepsilon)$. Our proofs rest on the following basic lemma.

Lemma 1. Let $F$ be a Montel subspace of $E$. Then there exist $n_{0} \in \mathbf{N}$ and $d>0$ such that, for every $x \in F$, we have

$$
r(x) \leq d r_{n_{0}}(x) \text {. }
$$

Proof. Suppose that (1) does not hold. Then, we have

$$
\forall n \in \mathbf{N}, \forall d>0, \exists x \in F \text { such that } r(x)>d r_{n}(x) .
$$

Let $\left(d_{n}\right)_{n}$ be an arbitrary sequence of positive numbers decreasing to 0 with $d_{1}<\frac{1}{2}$ and let $\tau_{n}=\left\|\left((1)_{k \leq n},(0)_{k>n}\right)\right\|$, for all $n \in \mathbf{N}$. Since $(\lambda,\|\|)$ satisfies the property $(\varepsilon)$ and we suppose that $(*)$ holds, we can find inductively a sequence $\left(x_{n}\right)_{n} \subset F, x_{n}=\left(x_{n k}\right)_{k}$, and a sequence of integers $1=k_{1}<k_{2}<\ldots<k_{n}<k_{n+1}<\ldots$ such that, for all $n \in \mathbf{N}$,

$$
\begin{gathered}
r\left(x_{n}\right)=1 \\
r_{k_{n}}\left(x_{n}\right)<\frac{d_{n}}{\tau_{\kappa_{n}}} \\
r\left(x_{n}-S_{k_{n+1}} x_{n}\right)<d_{n} .
\end{gathered}
$$


We claim that the closed linear span $\left[x_{n}: n \in \mathbf{N}\right] \subset F$ of $\left(x_{n}\right)_{n}$ in $E$ is an infinite dimensional Banach subspace of $F$ which leads to a contradiction because $F$ is a Montel space. To show this we proceed as follows.

Put $\bar{x}_{n}=\left(S_{k_{n+1}}-S_{k_{n}}\right)\left(x_{n}\right)$, for every $n \in \mathbf{N}$. Then, by (2) and $(\beta), r\left(\tilde{x}_{n}\right) \leq 1$. Also, by (3) and $(\beta), r\left(S_{k_{n}} x_{n}\right) \leq \tau_{k_{n}} r_{k_{n}}\left(S_{k_{n}} x_{n}\right)=\tau_{k_{n}} r_{k_{n}}\left(x_{n}\right)<d_{n}$ (by recalling that $\left\|f_{k}(x)\right\|_{k} \leq|x|_{k}$, for every $x \in X_{k}$ and for every $k \in \mathbf{N}$ ); also by (2) and (4) $r\left(S_{k_{n+1}} x_{n}\right)>1-d_{n}$. Hence, for every $n \in \mathbf{N}$, we have

$$
0<1-2 d_{1} \leq 1-2 d_{n}<r\left(\tilde{x}_{n}\right) \leq 1 .
$$

Moreover, by $(\beta)$, for every $j<i$ and $\left(a_{n}\right)_{n} \subset \mathbf{R}$, we obtain

$$
r\left(\sum_{n=1}^{i} a_{n} \tilde{x}_{n}\right) \leq r\left(\sum_{n=1}^{i} a_{n} \tilde{x}_{n}\right)
$$

hence, $\left(\tilde{x}_{n}\right)_{n}$ is a basic sequence with respect to the seminorm $r$ with basis constant $=1$.

Now, if $\tilde{x}=\sum_{n=1}^{\infty} a_{n} \tilde{x}_{n}$ converges with respect to $r$, then from (5) and (6) it follows that, for every $n \in \mathbf{N}$, we have

$$
r_{k_{i}}\left(\sum_{n=1}^{\infty} a_{n} \tilde{x}_{n}\right)=r_{k_{i}}\left(\sum_{n=1}^{i-1} a_{n} \tilde{x}_{n}\right) \leq \sum_{n=1}^{i-1}\left|a_{n}\right| r_{k_{i}}\left(\tilde{x}_{n}\right) \leq \frac{2 c_{i}}{1-2 d_{1}} r(\tilde{x})
$$

with $c_{i}=\sum_{n=1}^{i-1} r_{k_{i}}\left(\tilde{x}_{n}\right)<\infty$. Therefore, $\left(\tilde{x}_{k}\right)_{k}$ is a basic sequence of $E$ such that its closed span $\left[\tilde{x}_{n}: n \in \mathbf{N}\right]$ in $E$ is an infinite dimensional Banach subspace of $E$. We observe that from (2), (3), and (4) we then get for every $n \in \mathbf{N}$

$$
\begin{aligned}
r\left(x_{n}-\tilde{x}_{n}\right) & =\left\|\left(\left(\left\|f_{k}\left(x_{n k}\right)\right\|_{k}\right)_{k \leq k_{n}},(0)_{k_{n}<k \leq k_{n+1}},\left(\left\|f_{k}\left(x_{n k}\right)\right\|_{k}\right)_{k>k_{n+1}}\right)\right\| \\
& \leq\left\|\left(\left(\left\|f_{k}\left(x_{n k}\right)\right\|_{k}\right)_{k<k_{n}},(0)_{k>k_{n}}\right)\right\|+\left\|\left((0)_{k<k_{n+1}},\left(\left\|f_{k}\left(x_{n k}\right)\right\|_{k}\right)_{k>k_{n+1}}\right)\right\| \\
& \leq \tau_{k_{n}} r_{k_{n}}\left(x_{n}\right)+r\left(x_{n}-S_{k_{n+1}} x_{n}\right)<d_{n}+d_{n}=2 d_{n} .
\end{aligned}
$$

If we take $\left(d_{n}\right)_{n}$ small enough to have $d=\sum_{n=1}^{\infty} d_{n}<\frac{1-2 d_{1}}{4}$ (e.g., we can take $d_{n}=$ $\left.\left(\frac{1}{11}\right)^{n}\right)$, this implies that if $\tilde{x}=\sum_{n=1}^{\infty} a_{n} \tilde{x}_{n}$ converges, then $x=\sum_{n=1}^{\infty} a_{n} x_{n}$ also converges with respect to the seminorm $r$ and

$$
\left(1-\frac{4 d}{1-2 d_{1}}\right) r(\tilde{x}) \leq r(x) \leq\left(1+\frac{4 d}{1-2 d_{1}}\right) r(\tilde{x}) .
$$

Finally, from (3), (7) and (8) it follows that, for every $i \in \mathbf{N}$,

$$
\begin{aligned}
r_{k_{i}}(x) & \leq r_{k_{i}}\left(\sum_{n=1}^{i-1} a_{n} x_{n}\right)+r_{k_{i}}\left(\sum_{n \geq i} a_{n} x_{n}\right) \\
& \leq \sum_{n=1}^{i-1}\left|a_{n}\right| r_{k_{i}}\left(x_{n}\right)+\sum_{n \geq i}\left|a_{n}\right| r_{k_{i}}\left(x_{n}\right) \\
& \leq \frac{2}{1-2 d_{1}} r(\tilde{x})\left(\sum_{n=1}^{i-1} r_{k_{i}}\left(x_{n}\right)+\sum_{n \geq i} \frac{d_{n}}{r_{k_{n}}}\right) \\
& \leq \frac{2 c_{i}^{\prime}}{1-6 d} r(x),
\end{aligned}
$$


with $c_{i}^{\prime}=\sum_{n=1}^{i-1} r_{k_{i}}\left(x_{n}\right)+\sum_{n \geq i} \frac{d_{n}}{\tau_{k_{n}}}<\infty$. (We may always assume that $\tau_{n} \geq 1$ for every $n$.) This and (8) imply that $\left(x_{n}\right)_{n}$ is a basic sequence of $E$ equivalent to $\left(\tilde{x}_{n}\right)_{n}$, so that $\left[x_{n}: n \in \mathbf{N}\right]$ is an infinite dimensional Banach subspace of $F$. This completes the proof.

Consequently, we obtain the following result.

THEOREM 2. Let $F$ be a Montel subspace of $E$. Then the topology induced on $F$ by $E$ coincides with the one induced by $\prod_{k=1}^{\infty} X_{k}$.

Remarks. 1. By Theorem 2, to construct some examples of Montel subspaces $F$ of a given Fréchet space $E$ of Moscatelli type it suffices to look at the Montel subspaces of $\prod_{k=1}^{\infty} X_{k}$ for which the only algebraic condition $\left(f_{k}\left(x_{k}\right)\right)_{k} \in \lambda\left(\left(Y_{k},\|\|_{k}\right)\right.$ is satisfied, for every $x=\left(x_{k}\right)_{k} \in F$.

2. If we suppose that $E$ is a Montel space, then from Theorem 2 it follows that $E=\prod_{k=1}^{\infty} X_{k}$. This and inequality (1) imply that $\operatorname{dim} X_{k}<\infty$ for every $k \in \mathbf{N}$ and there exists $k_{0}$ such that $f_{k}\left(X_{k}\right)=\{0\}$, for every $k \geq k_{0}$. Therefore, Proposition 2.7 of [4] can be seen as a particular case of Theorem 2 .

Now we can state and prove our main result.

THEOREM 3. If $F$ is a complemented Montel subspace of $E$, then either $F=\omega$ or $\operatorname{dim} F<\infty$.

Proof. Suppose that $F$ is a complemented Montel subspace of $E$ and $P: E \rightarrow F$ is a projection with $\operatorname{ker} P=G$ and $Q=I-P$. Now, by Theorem $2, F$ is a Montel subspace of $\prod_{k=1}^{\infty} X_{k}$. Therefore, by Lemma 1.1 of [6], $F=\operatorname{proj}_{n}\left(S_{n}(F), \tilde{S}_{n+1, n}\right)$, where $\tilde{S}_{n+1, n}: S_{n+1}(F) \rightarrow S_{n}(F)$, restrictions of the $S_{n+1, n}$ 's, are clearly surjective. We shall show that $S_{n}(F)$ is a closed subspace of $\prod_{k \leq n} X_{k}$ and hence it is a Banach space. Consequently, $F$ is a quojection; for the definition see $[8,8.4 .27]$. Since $F$ is Montel, it must be either isomorphic to $\omega$ or finite dimensional; see, e.g., $[8,8.4 .31]$. Then, we put $H_{n}=S_{n}(F) \subset$ $\prod_{k \leq n} X_{k}$ and we denote by $\bar{H}_{n}$ its closure in $\prod_{k \leq n} X_{k}$, for every $n \in \mathbf{N}$. Moreover, we denote again by $J_{n}$ and $\bar{J}_{n}$ the restriction of $J_{n}$ to $H_{n}$ and $\bar{H}_{n}$ respectively. Since $F$ is Montel, the composition maps

$$
\begin{aligned}
& P_{n}=S_{n} P J_{n}: H_{n} \stackrel{J_{n}}{\longrightarrow} E \stackrel{P}{\longrightarrow} F \stackrel{S_{n}}{\longrightarrow} H_{n}, \\
& \bar{P}_{n}=S_{n} P \bar{J}_{n}: \bar{H}_{n} \stackrel{\bar{J}_{n}}{\longrightarrow} E \stackrel{P}{\longrightarrow} F \stackrel{S_{n}}{\longrightarrow} H_{n}
\end{aligned}
$$

are compact for every $n \in \mathbf{N}$, where $\bar{P}_{n}$ is the compact extension of $P_{n}$ to $\bar{H}_{n}$. Now, we observe that if $x \in H_{n}$, then $J_{n} x=P J_{n} x+Q J_{n} x$, and hence $x=S_{n} P J_{n} x+S_{n} Q J_{n} x$ (recalling that $J_{n}$ is a right-continuous inverse of $S_{n}$ ). This implies that $S_{n} Q J_{n} x=x-S_{n} P J_{n} x=$ $x-P_{n} x$ belongs to $H_{n}$ too. Therefore, the composition map

$$
Q_{n}=S_{n} Q J_{n}: H_{n} \stackrel{J_{n}}{\longrightarrow} E \stackrel{Q}{\longrightarrow} G \stackrel{S_{n}}{\longrightarrow} S_{n}(G)
$$


is such that its range $Q_{n}\left(H_{n}\right)$ is also contained in $H_{n}$. Clearly, $I_{H_{n}}=P_{n}+Q_{n}$ and so $Q_{n}=I_{H_{n}}-P_{n}$. Since $P_{n}$ is a compact map from $H_{n}$ into itself, it follows that $Q_{n}\left(H_{n}\right) \subset H_{n}$ is a closed subspace of $H_{n}$ and hence it is a Banach subspace of $\prod_{k \leq n} X_{k}$. This implies that $\bar{Q}_{n}=S_{n} Q \bar{J}_{n}$, which is the continuous extension of $Q_{n}$ to $\bar{H}_{n}$, is such that its range $\bar{Q}_{n}\left(\bar{H}_{n}\right)$ is also contained in $H_{n}$. Now, we can prove that $H_{n}$ is a closed subspace of $\prod_{k \leq n} X_{k}$. In fact, if $\left(x_{j}\right)_{j} \subset H_{n}$ and $\left(x_{j}\right)_{j}$ converges to $x \in \bar{H}_{n}$ in $\prod_{k \leq n} X_{k}$, then $\left(P_{n} x_{j}\right)_{j}$ converges to $\bar{P}_{n} x \in H_{n}$ and $\left(Q_{n} x_{j}\right)_{j}$ converges to $\bar{Q}_{n} x \in H_{n}$. However $x_{j}=P_{n} x_{j}+Q_{n} x_{j}$, for every $j$, and hence, letting $j \rightarrow \infty$, we obtain $x=\bar{P}_{n} x+\bar{Q}_{n} x \in H_{n}$. Thus, the proof is complete.

COROLLARY 4. If $E$ is a Fréchet space of Moscatelli type with a continuous norm, then $E$ does not have an infinite dimensional complemented Montel subspace.

3. Applications to Fréchet-Sobolev spaces. Let $\Omega$ be an open subset of $\mathbf{R}^{N}$, with $N \geq 1$. For $m, k \in \mathbf{N}, k \leq m$ and $1 \leq p<\infty, C^{m}(\Omega) \cap H^{k, p}(\Omega)$ is a Fréchet space with its natural intersection topology given by the sequence of norms

or

$$
p_{n}(f)=\max _{|\alpha| \leq k}\left(\int_{\Omega}\left|f^{(\alpha)}(x)\right|^{p} d x\right)^{1 / p}+\max _{|\alpha| \leq m} \max _{x \in J_{n}}\left|f^{(\alpha)}(x)\right| \text {, for } p<\infty,
$$

$$
p_{n}(f)=\max _{|\alpha| \leq k} \sup _{x \in \Omega}\left|f^{(\alpha)}(x)\right|+\max _{|\alpha| \leq m} \max _{x \in J_{n}}\left|f^{(\alpha)}(x)\right| \text {, for } p=\infty,
$$

where $\left(J_{n}\right)_{n}$ is a sequence of compact subsets of $\Omega$ such that $J_{n}=\bar{J}_{n} \subset \mathcal{J}_{n+1}$ and $\cup_{n} J_{\dot{n}}=\Omega$. Moreover, for $1 \leq q<p \leq \infty$, the space $L_{\mathrm{oc}}(\Omega) \cap L^{q}(\Omega)$ of all $q$-integrable and locally $p$-integrable functions in $\Omega$ is also a Fréchet space, whose topology is given by the following sequence of norms

or

$$
q_{n}(f)=\left(\int_{\Omega}|f(x)|^{q} d x\right)^{1 / q}+\left(\int_{J_{n}}|f(x)|^{p} d x\right)^{1 / p}, \text { for } p<\infty,
$$

$$
q_{n}(f)=\left(\int_{\Omega}|f(x)|^{q} d x\right)^{1 / q}+\sup _{x \in J_{n}}|f(x)|, \text { for } p=\infty,
$$

where $\left(J_{n}\right)_{n}$ is a sequence of compact subsets of $\Omega$ defined as above. Now, by Theorems 1 and 2 of [2] (see also [3]), the Fréchet space $C^{m}(\Omega) \cap H^{k, p}(\Omega)$ is of Moscatelli type for $k=0,1$ when $\Omega$ is an open subset of $\mathbf{R}^{N}(N>1)$ and for every $k \in \mathbf{N}$ when $\Omega$ is an open subset of $\mathbf{R}$ or $\Omega=\mathbf{R}^{N}$. Also, the Fréchet space $L_{\mathrm{loc}}(\Omega) \cap L^{q}(\Omega), 1 \leq q<p \leq \infty$, is of Moscatelli type, as it follows from the fact that the map

$$
\begin{aligned}
L_{\mathrm{oc}}^{p_{0}}(\Omega) \cap L^{q}(\Omega) & \rightarrow\left(\prod_{n=1}^{\infty} L^{p}\left(K_{n}\right)\right) \cap l^{q}\left(L^{q}\left(K_{n}\right)\right) \\
f & \rightarrow\left(f_{\mid K_{n}}\right)_{n},
\end{aligned}
$$

where $K_{1}=J_{1}$ and $K_{n+1}=\overline{J_{n+1} \backslash J_{n}}$, for every $n \geq 1$, is an isomorphism onto. Finally, we recall that the inclusion map $H^{m p}(\Omega) \hookrightarrow L^{q}(\Omega)$ is continuous if $m \in \mathbf{N}, 1 \leq p<q<\infty$, and $N-m p>0, \frac{m}{N} \geq \frac{1}{p}-\frac{1}{q}>0$ and if $\Omega$ is an open subset of $\mathbf{R}^{N}$ with the cone property, by the 
Sobolev imbedding theorem. (See, for example, Theorem 5.4 of [1]). Then, the space $H_{\mathrm{loc}}^{m, p}\left(\mathbf{R}^{N}\right) \cap L^{q}\left(\mathbf{R}^{N}\right)$, with $m, p$ and $q$ satisfying the above conditions, is a Fréchet space with respect to the topology generated by the following sequence of norms

$$
r_{n}(f)=\max _{|\alpha| \leq m}\left(\int_{\left\{x \in \mathbf{R}^{N}:|x| \leq n\right\}}\left|f^{\alpha}(x)\right|^{p} d x\right)^{1 / p}+\left(\int_{\mathbf{R}^{N}}|f(x)|^{q} d x\right)^{1 / q} .
$$

The same proof of Theorem 1 of [2] works to show that the spaces $H_{\mathrm{loc}}^{m p}\left(\mathbf{R}^{N}\right) \cap L^{q}\left(\mathbf{R}^{N}\right)$ are of Moscatelli type.

Then, by Theorems 2 and 3 of $\$ 2$, we can deduce the following results.

COROLLARY 5. Let $E$ be one of the following three spaces:

(a) $C^{m}(\Omega) \cap H^{k, p}(\Omega)$, with $k=0,1$ when $\Omega$ is an open subset of $\mathbf{R}^{N}(N>1)$ or $k \in \mathbf{N}$ when $\Omega$ is an open subset of $\mathbf{R}$ or $\Omega=\mathbf{R}^{N}$, with $m \in \mathbf{N}, m \geq k$, and $1 \leq p<\infty$;

(b) $L_{\mathrm{loc}}^{p}(\Omega) \cap L^{q}(\Omega)$, with $\Omega$ an open subset of $\mathbf{R}^{N}(N \geq 1)$ and $1 \leq q<p \leq \infty$;

(c) $H_{\mathrm{luc}}^{m, p}\left(\mathbf{R}^{N}\right) \cap L^{q}\left(\mathbf{R}^{N}\right)$, with $m \in \mathbf{N}, 1 \leq p<q<\infty$, and $N-m p>0, \frac{m}{N}>\frac{1}{p}-\frac{1}{q}>0$; and let $F$ be a Montel subspace of $E$. Then the topology induced on $F$ by $E$ coincides with the one induces by $C^{m}(\Omega)$, by $L_{\mathrm{loc}}^{p_{\mathrm{c}}}(\Omega)$ and by $H_{\mathrm{loc}}^{m p}\left(\mathbf{R}^{N}\right)$ in the case $(\mathrm{a}),(\mathrm{b})$ and $(\mathrm{c})$ respectively.

COROLlary 6. The Fréchet spaces considered in Corollary 5 do not have an infinite dimensional complemented Montel subspace.

Remark 3. We note that Corollaries 5 and 6 remain valid also for $C^{m}(\Omega) \cap H^{k s}(\Omega)$ with $k>1$ and $\Omega$ an arbitrary open subset of $\mathbf{R}^{N}$, with $N>1$, although a representation of type (0) is not available. The proof in this case is similar to the proofs of Theorems 2 and 3 of $\$ 2$ with some simple changes.

\section{REFERENCES}

1. R. A. Adams, Sobolev spaces (Academic Press, London 1970).

2. A. A. Albanese, G. Metafune and V. B. Moscatelli, Representations of the spaces $C^{m}(\Omega) \cap H^{k, p}(\Omega)$, Math. Proc. Camb. Phil. Soc., $120(1996), 489-498$.

3. A. A. Albanese, G. Metafune and V. B. Moscatelli, Representations of the spaces $C^{m}\left(\mathbf{R}^{N}\right) \cap H^{k,}\left(\mathbf{R}^{N}\right)$, in Proceedings of the First Workshop of Functional Analysis at Trier University, (Walter de Gruyter and Co., 1996), 11-20.

4. J. Bonet, S. Dierolf, Fréchet spaces of Moscatelli type, Rev. Mat. Univ. Complut. Madrid 2 (suppl.) (1989), 77-92.

5. H. Jarchow, Locally convex spaces (Teubner, Stuttgart, 1981).

6. G. Metafune and V. B. Moscatelli, Complemented subspaces of sums and products of Banach spaces, Ann. Mat. Pura Appl. 153 (4) (1988), 175-190.

7. V. B. Moscatelli, Fréchet spaces without continuous norms and without basis, Bull. London Math. Soc. 12 (1980), 63-66.

8. P. Pérez Carreras and J. Bonet, Barrelled locally convex spaces (North Holland Math. Studies 131, 1987).

Dipartimento Di Matematica

UNIVERSITȦ DI LECCE, C.P. 193

Via Per Arnesano

73100, LeCCE, ITALY 